\title{
Subxiphoid uniportal lobectomy
}

\author{
Mohamed M. Moneer ElSaegh ${ }^{1}$, Nur Aziah Ismail ${ }^{2}$, Mohamed I. Mydin ${ }^{1}$, Marco Nardini $^{1}$, Joel Dunning ${ }^{1}$ \\ ${ }^{1}$ Department of Cardiothoracic Surgery, the James Cook University Hospital, Middlesbrough, UK; ${ }^{2}$ Department of Cardiothoracic Surgery, National \\ heart institute (IJN), Kuala Lumpur, Malaysia \\ Correspondence to: Mohamed M. Moneer ElSaegh. Department of Cardiothoracic Surgery, the James Cook University Hospital, Middlesbrough, UK. \\ Email: mmmoneer@hotmail.com.
}

\begin{abstract}
Video-assisted thoracic surgery (VATS) surgery has seen an evolution from multiple ports to uniportal and finally subxiphoid uniportal recently. In traditional VATS surgery, the instruments and the thoracoscope enter the thoracic cavity through two to four operating ports on the lateral chest wall, which can cause chronic pain and chest wall numbness. However single-portal VATS surgery could potentially cause similar problems as the port is placed in between the ribs. In March 2015 Liu et al. reported a VATS bilateral pulmonary metastasectomy and right middle lobectomy via a subxiphoid uniportal technique. The advantage of the uniportal subxiphoid approach is the ability to use different size of instruments and freedom of movement as there is no limitation by the ribs. Post-operative pain typically experienced due to bruising of the intercostal nerves is also avoided in this approach. Shanghai Pulmonary hospital has taken VATS surgery to the next level with subxiphoid uniportal VATS (SVATS) lung resection, whereby this method is performed in large volumes of cases. Here we describe our experience of a uniportal subxiphoid VATS right middle lobectomy using the Shanghai technique, the first in the UK. A uniportal sub-xiphoid lobectomy was performed on a 62-year-old lifelong smoker male patient with a histological diagnosis of right middle lobe adenocarcinoma, measuring $1.5 \mathrm{~cm}$ and radiological staging of T1aN0M0. We have been performing microlobectomies in our institution (with the utility port placed in the subxiphoid region) which is technically similar to this approach. This is the first subxiphoid uniportal lobectomy performed in the UK. The operation was done successfully and the patient was discharged home 2 days later without any complications.
\end{abstract}

Keywords: Video-assisted thoracic surgery (VATS); minimally invasive; micro lobectomy; subxiphoid

Received: 17 October 2016; Accepted: 10 December 2016; Published: 08 March 2017.

doi: 10.21037/jovs.2016.12.05

View this article at: http://dx.doi.org/10.21037/jovs.2016.12.05

\section{Introduction}

Video-assisted thoracic surgery (VATS) surgery has seen an evolution from multiple ports to uniportal and finally subxiphoid uniportal recently. The assumed benefits from VATS over open technique includes: shorter hospital stay, less postoperative pain, cosmesis, less blood loss, earlier resumption of daily activities, better tolerance to postoperative chemotherapy (1-9). In traditional VATS surgery, the instruments and the thoracoscope enter the thoracic cavity through two to four operating ports on the lateral chest wall, which can cause chronic pain and chest wall numbness. Single-portal VATS surgery could potentially cause similar problems as the port is placed in between the ribs. Liu et al. reported a subxiphoid uniportal VATS (SVATS) bilateral pulmonary metastasectomy and right middle lobectomy in March 2015 (1). The advantage of the uniportal subxiphoid approach is the ability to use different size of instruments and freedom of movement as there is no limitation by the ribs. In Shanghai Pulmonary hospital VATS surgery was taken to another level by performing large volumes of lung resections via subxiphoid uniportal approach.

A 62-year-old lifelong smoker presented with a right middle lobe mass measuring $1.5 \mathrm{~cm}$ diameter. Histological diagnosis of adenocarcinoma was made and radiological 


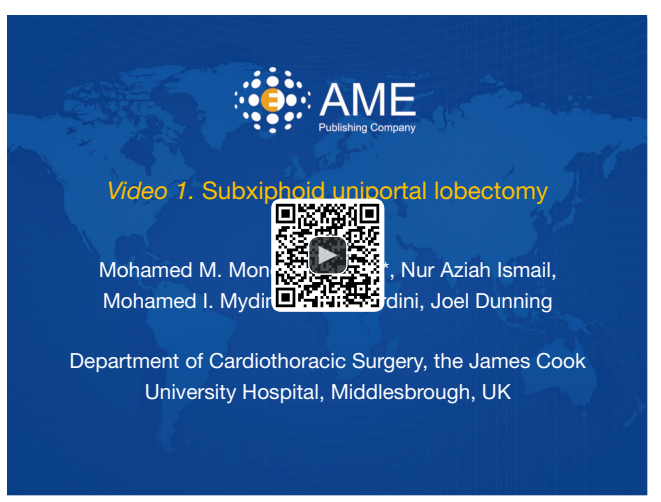

Figure 1 Subxiphoid uniportal lobectomy (10).

Available online: http://www.asvide.com/articles/1384

staging of the tumour was T1aNoMo. The patient underwent uniportal subxiphoid right middle lobectomy and was discharged home with 2 days post-operatively without any complications.

\section{Patient selection and workup}

Patient selection for subxiphoid only VATS lobectomy is the same as typical VATS lobectomy.

Resectability of the tumor could be assessed by performing CT scan of the chest. CT guided biopsy or endobronchial ultrasound biopsy is helpful in providing pre-operative histology. Distant metastasis should be ruled out via PET scan and CT head.

Fitness for surgery could be assessed by lung function test and CPEX, as well as routine bloods (FBC, U\&E, and coagulation profile).

\section{Pre-operative preparation}

Following the above, all patients should have preoperative blood test and baseline chest X-ray. Cervical mediastinoscopy as per European guidelines. ECG is done to rule out heart disease (9).

\section{Equipment preference card}

* $\mathrm{CO}_{2}$ insufflation is helpful in pushing the diaphragm downwards hence giving plenty of room for maneuvering during the operation;

* Standard staplers and VATS instruments are used via the subxiphoid incision;

* Microcutter ${ }^{\mathrm{TM}}$ stapler, endodissector and a wide range of
$5 \mathrm{~mm}$ VATS instruments including camera are optional (9).

\section{Procedures}

Patient is positioned on a left lateral decubitus, to achieve good access to the sub-xiphoid space and to provide us with the option of adding more ports if required (Figure 1).

A $5 \mathrm{~cm}$ skin incision is made in the midline just under the xiphoid process. The Linea Alba is opened and xiphoid process is excised to provide more space for this subxiphoid uniportal approach. Dissection is made all the way down until chest cavity is entered. Next, a Langenbeck on a right angle stand is used to retract open the port laterally. One end of the Alexis port is inserted into a $10 \mathrm{~mL}$ port without the trocar, and this $10 \mathrm{ml}$ port is then inserted into the chest cavity. Keeping the other end of the Alexis port out of the chest, a trocar is inserted into the $10 \mathrm{ml}$ port to expel the other end of the Alexis port into the chest.

Dissection of the inferior pulmonary ligament is then carried out to mobilize the lung. The middle lobe is identified and tumour is checked, any adhesions is taken down using hook diathermy. Middle lobe pulmonary veins are then located and exposed, followed by exposure of the pulmonary artery. Any lymph node in the way is taken out at this point to free up the structures and therefore allowing freedom of movement.

A $5 \mathrm{~mL}$ Sils dissector is used to go around the middle lobe pulmonary vein, followed by a Microcutter ${ }^{\mathrm{TM}}$ to staple the vein. A lymph node grasper is then used to develop the oblique fissure; the anterior part of the oblique fissure is then completed with the purple tri-stapler. Middle lobe bronchus is then located and a sling is placed around the bronchus. The lymph node grasper is used to create a plane behind the bronchus and a purple tristapler is then railroaded around the bronchus. Once the bronchus is stapled, a great view of the segmental pulmonary artery is achieved. A $5 \mathrm{ml}$ endodissector with a 60 -degree angle is then used to go around the pulmonary artery branches and divided with the Microcutter ${ }^{\mathrm{TM}}$ at an 80-degree angle. Finally the fissure is completed with multiple firings of purple tristapler. The lung is placed in an endobag prior to removal from the chest to prevent seeding of tumours at the port site (11).

Lymphadenectomy of station $4 \mathrm{R}$ and $2 \mathrm{R}$ is done using lymph node dissector and hook diathermy. To perform the station 7 lymphadenectomy, the lower lobe is retracted forward and the spine is located, the upper lobe is retracted forward and the bronchus intermedius is located. The pleura are dissected with hook diathermy and station 7 is resected. 
After haemostasis is carried out, an extrapleural catheter $\left(\mathrm{ON}-\mathrm{Q}\right.$ Tunneler $\left.{ }^{\circledR}\right)$ is placed up from the $8^{\text {th }}$ rib up to the $3^{\text {rd }}$ intercostal space. A chest drain is placed via the subxiphoid port prior to closure.

A self-retaining retractor is used during closure of the subxiphoid port in order to obtain a good view of the linea alba and the rectus abdominis.

\section{Role of team members}

All lung cancer patients are discussed in the Lung cancer multi-disciplinary team (MDT) meeting to reach a consensus about the best modality of treatment to be offered to each patient. Selected surgical patients are then reviewed in the outpatient clinic by thoracic surgeons and treatment options are discussed in details. Pre-operative assessment is run by the thoracic specialist nurses in parallel, for general examination of the patients and to ensure that all relevant investigations i.e., blood tests, CT scan, PET scan, etc. are in place and up to date.

On admission the patient and all investigations are reviewed again by the thoracic team. Our policy is to have the CT scan within 6 weeks of surgery date, this is to ensure that the tumor has not either spread or become inoperable.

Patients are seen by the anesthetist on admission, to ensure they are fit for surgery and to ascertain whether special requirement is needed for intubation i.e., previous head and neck surgery.

Immediately prior to the operation the anesthetist inserts arterial and central venous line. Double lumen endotracheal tube is used for intubation to achieve single lung ventilation for the lobectomy. Intra-operative monitoring of the patient is achieved through ECG, arterial line pressure monitoring, end tidal $\mathrm{CO}_{2}$ level and pulse oximetry.

It is crucial that the surgeon is present in the anesthetic room during the intubation and positioning of the patient to ensure that satisfactory single lung ventilation could be achieved and the patient is positioned correctly for the operation.

The anesthetist checks that the patient is pain free in the immediate post-operative period, in the recovery room and the high dependency unit (HDU). For VATS Lobectomy we ensure our patients receive IV patient controlled analgesia (PCA).

To rule out any immediate post-operative complications, all patients will have a post-op chest X-ray which is checked by the surgical registrars. Daily review of the patient, pain score, chest drain and chest $\mathrm{X}$-ray is carried out by a team of specialist nurses and surgical registrar and the operating surgeon.

Physiotherapist plays a crucial role in the post-operative management to ensure early mobility and prevent respiratory complications. Deep breathing techniques and incentive spirometry are provided by the physiotherapists to patients.

After discharge from the hospital, patients are reviewed in the outpatient clinic by either the surgeon or specialist nurse after 6 weeks, where they have a chest X-ray, surgical wounds are checked, post-operative histology and staging and result of the MDT discussion of the pathological staging (whether a chemotherapy/radiotherapy is required) is then delivered to the patient (9).

\section{Post-operative management}

Routine post VATS lobectomy management with $20 \mathrm{Fr}$ intercostal drains attached to under water seal. Post-operative chest $\mathrm{X}$-ray is done to ensure there is no early complication. In this case we removed the chest drain on day 1 postoperative day. Intravenous PCA was used immediately post-op and converted to oral analgesia once the drain is removed. Early mobilization and adequate analgesia is key in the post-operative management of lung resection. (9).

\section{Tips, tricks and pitfalls}

* Special attention should be paid during the closure of the subxiphoid port in order to prevent incisional hernia especially in overweight/obese patients. It is important to get an excellent view of the linea alba and rectus abdominis muscle on both sides (we suggest using a self-retaining retractors) and to incorporate these in your closing sutures, taking deep bites on both sides especially in the linea alba;

* To prevent diaphragmatic herniation, dissect medially under the sternum on entering the chest cavity, to prevent stripping of the diaphragm laterally of the inferior costal margin;

* The advantage of subxiphoid only approach is the option of using different size of instruments i.e., not limited to only $5 \mathrm{~mL}$ instruments and a wider range of movements with the instruments as there is no limitation by the ribs;

* When completing the fissure, ensure that you can see above and below at all times and be generous with the margin;

* Contrary to the common belief, lymphadenectomy can 
be easily performed using this approach, especially with the help of 30 degree camera and reticulating/angulating instruments.

\section{Acknowledgements}

None.

\section{Footnote}

Conflicts of Interest: The authors have no conflicts of interest to declare.

Informed Consent: Written informed consent was obtained from the patient for publication of this manuscript and any accompanying images.

\section{References}

1. Liu CC, Wang BY, Shih CS, et al. Subxyphoid singleincision thoracoscopic pulmonary metastasectomy. Thorac Cancer 2015;6:230-2.

2. Hernandez-Arenas LA, Lin L, Yang Y, et al. Initial experience in uniportal subxiphoid video-assisted thoracoscopic surgery for major lung resections. Eur J Cardiothorac Surg 2016. [Epub ahead of print].

3. Whitson BA, Andrade RS, Boettcher A, et al. Videoassisted thoracoscopic surgery is more favorable than thoracotomy for resection of clinical stage I non-small cell

doi: 10.21037/jovs.2016.12.05

Cite this article as: ElSaegh MM, Ismail NA, Mydin MI, Nardini M, Dunning J. Subxiphoid uniportal lobectomy. J Vis Surg 2017;3:24. lung cancer. Ann Thorac Surg 2007;83:1965-70.

4. Shigemura N, Akashi A, Funaki S, et al. Long-term outcomes after a variety of video-assisted thoracoscopic lobectomy approaches for clinical stage IA lung cancer: a multi-institutional study. J Thorac Cardiovasc Surg 2006;132:507-12.

5. Sugiura H, Morikawa T, Kaji M, et al. Long-term benefits for the quality of life after video-assisted thoracoscopic lobectomy in patients with lung cancer. Surg Laparosc Endosc Percutan Tech 1999;9:403-8.

6. Nicastri DG, Wisnivesky JP, Litle VR, et al. Thoracoscopic lobectomy: report on safety, discharge independence, pain, and chemotherapy tolerance. J Thorac Cardiovasc Surg 2008;135:642-7.

7. Sahai RK, Nwogu CE, Yendamuri S, et al. Is thoracoscopic pneumonectomy safe? Ann Thorac Surg 2009;88:1086-92.

8. ElSaegh MM, Ismail NA, Gordon J, et al.

Micropneumonectomy (pneumonectomy using the videoassisted thoracic surgery microlobectomy technique).

Asvide 2016;3:216. Available online: http://www.asvide. com/articles/973

9. ElSaegh MM, Ismail NA, Gordon J, et al. Video-assisted thoracic surgery micro pneumonectomy, a new approach. J Vis Surg 2016;2:94.

10. ElSaegh MM, Ismail NA, Mydin MI, et al. Subxiphoid uniportal lobectomy. Asvide 2017;4:077. Available online: http://www.asvide.com/articles/1384

11. Thurer RL. Video-assisted thoracic surgery. Ann Thorac Surg 1993;56:199-200. 